\title{
Laboratory Data Domain
}

National Cancer Institute

\section{Source}

National Cancer Institute. Laboratory Data Domain. NCI Thesaurus. Code C49592.

A subject domain utilized for the submission of information encompassing and representing data, vocabulary or records related to laboratory data. 